QoS- Aware and Load-Bal ance Rout i ng for I EEE 802. 11s Based Nei ghbor hood Ar ea Net work i n Smart Grid

\begin{tabular}{|l|l|}
\hline 著者 & $\begin{array}{l}\text { DENG Xi aoheng, HE Li fang, ZHU Congxu, DONG } \\
\text { M anxi ong, OTA Kaor u, CAl Li n }\end{array}$ \\
\hline $\begin{array}{l}\text { j our nal or } \\
\text { publ i cat i on t i t l e }\end{array}$ & W r el ess Per sonal Communi cat i ons \\
\hline vol une & 89 \\
\hline number & 4 \\
\hline page r ange & $1065-1088$ \\
\hline year & $2016-08$ \\
\hline URL & ht t p: //hdl . handl e. net /10258/00009014 \\
\hline
\end{tabular}




\title{
QoS-aware and Load-balance Routing for IEEE 802.11s based Neighborhood Area Network in Smart Grid
}

\author{
Xiaoheng Deng ${ }^{1}$, Lifang $\mathrm{He}^{1}$, Congxu Zhu ${ }^{1}$, Mianxiong Dong ${ }^{2}$, Kaoru Ota ${ }^{2}$, Lin $\mathrm{Cai}^{3}$ \\ ${ }^{1}$ School of Information Sci \& Eng, Central South University, Changsha 410083, China \\ ${ }^{2}$ Department of Information and Electronic Engineering, Muroran Institute of Technology, \\ Muroran 050-8585, Japan \\ ${ }^{3}$ Department of Electrical and Computer Engineering, University of Victoria, Victoria V8P \\ 5C2, Canada \\ Corresponding Author: Mianxiong Dong \\ mx.dong@csse.muroran-it.ac.jp \\ Tel: +81-143-46-5473
}

\begin{abstract}
Monitoring and transforming smart grid (SG) assets in a timely manner is highly desired for emerging smart grid applications. This critically requires the design of a neighborhood area network (NAN) which is capable of providing high-efficiency and reliable two-way last mile communication from meters to other SG domains. For this demand, IEEE 802.11s based wireless mesh network (WMN) is anticipated to be utilized in a NAN as it can provide high scalability, high-speed and cost-effective wireless transmission. In this paper, we propose a NAN QoS-aware and load-balance routing scheme (NQALB) based on the default hybrid wireless mesh protocol (HWMP) of IEEE 802.11s, which aims to address multiple QoS requirements from different NAN applications, and guarantee the highly reliability transfer of NAN traffic data towards gateway. With the NQA-LB, various QoS requirements can be satisfied through sufficient differentiated services as well as network congestion is mitigated by achieving load balance between multiple transmission paths. In order to improve the reliability of NQA-LB, we present an EDCA based adaptive priority adjustment scheme, called AP-EDCA, which dynamically adjusts packet's priority to increase the throughput under low load condition and to mitigate the collision under heavy load condition to improve the reliability of applications with high QoS requirements. Extensive simulation experiments demonstrate the superiority of the proposed scheme in terms of packet delivery ratio, end-to-end delay and throughput while satisfies various QoS requirements much better at the same time.
\end{abstract}

Keywords: smart grid, neighborhood area network (NAN), wireless mesh network (WMN), IEEE 802.11s, EDCA

\section{INTRODUCTION}

Establishing reliable and real-time bi-directional communication system is vital for smart grid (SG) to work efficiently. Through bi-directional communication system, 
customers can optimize their electricity consumption for minimizing utility costs. Furthermore, the control centers can make real-time power pricing and many other decisions according to energy demands to improve the resource utilization [1, 2]. Hence, a balance of power generation and demands in the entire grid can be achieved to significantly improve power quality and efficiency by bi-directional communication system. According to National Institute of Standards and Testing (NIST) [3], SG communication infrastructure consists of three different networks. These networks are home area network (HAN), neighborhood area network (NAN) and wide area network (WAN). A HAN focuses on small-scale data communication between devices inside typical households. A NAN is defined to provide a backbone for data that are transmitted from multiple HANs as well as providing various services of its own. While a WAN connects grid control centers and NAN data concentrators with long distance in very large areas, and transmits data in a very high-speed manner. These networks could utilize any appropriate communication technology such as broadband power line communications (BPLC), wireless sensor networks (WSNs), wireless local area networks (WLANs), and wireless mesh networks (WMNs), as they should provide different functional requirements [2]. In this paper, we specially focus on NAN system which forms data transmission bridge between WAN and HAN in SG.

As one of the core technologies, an efficient and reliable communication network plays an important role in realization of all the goals of SG NAN [4]. NAN collects the electricity utility data from multiple HANs and forwards it to backbone through NAN gateways. Meanwhile, it delivers control and pricing messages in the reverse direction. Some data are transmitted in a periodic manner such as periodic metering and periodic power quality data. While some other data must be transmitted in a timely manner with high QoS, such as demand response (DR) is considered as a killer application for smart grid that even some studies are dedicated to develop reliable and efficient wireless communication networks especially for DR [5,6]. These varieties of traffic characteristics that lead to NAN require high standard of reliable and various QoS transmission technology to support various electrical services and applications. The design of such a network depends not only on the application layer requirement, but also on the nature of its medium access control (MAC) and physical (PHY) layer. Since WMN supports various physical modules including $802.11 \mathrm{a} / \mathrm{b} / \mathrm{g} / \mathrm{n}$, it is permissible to handle various applications such as bulk of data and time stringent data. In addition, WMN is automatically self-organized and self-healed when network problems occur so that it is useful to establish robust connections. Furthermore, it also provides cost-effective deployment, easy network maintenance and reliable service coverage [7]. Therefore, WMN technology which is based on IEEE 802.11s stands [8] can be a potential candidate for reliable and high-speed wireless NANs applications. And some unique functions of IEEE 802.11s are also expected to support various QoS requirements of NAN especially [13]. Firstly, the root to mesh STAs (stations) topology provides an ideal topology for NANs. For example, the upstream data (such as AMI data, power quality monitoring, and video surveillance) generated by NAN meters can be constantly transmitted to root that forwards the data to a head-end in SG, the structure of IEEE 802.11s WMN for NAN is shown in Fig. 1. Secondly, the default hybrid wireless mesh protocol (HWMP) of IEEE 802.11s suggests a hybrid rout- 
ing rule and airtime link metric (ALM) to suit for static WMNs in smart grid NANs. Finally, IEEE 802.11s adopts the enhanced distributed coordination access (EDCA) scheme from IEEE 802.11e [9] for various QoS provisioning by utilizing access categories (ACs) mapped into multiple data queues, which is also a representative requirement in NANs.

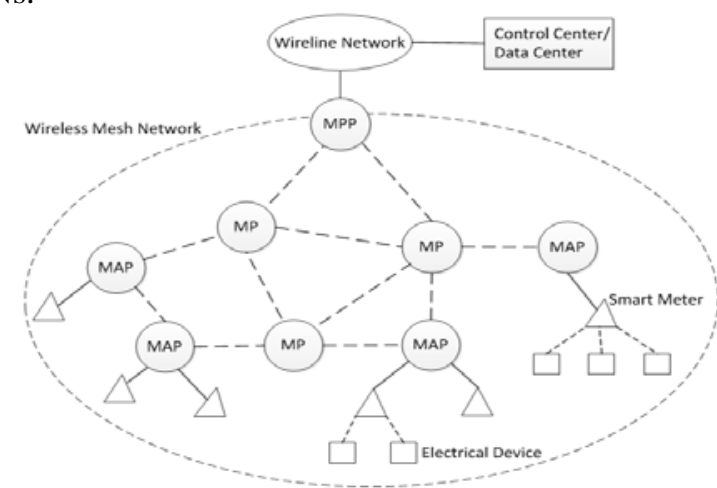

Fig. 1. IEEE 802.11s WMN for NAN in SG

However, some new challenges can be anticipated when the original IEEE 802.11s is applied to the smart grid NAN. The first challenge is that the default ALM in HWMP defines the fixed length test frame and the same MAC retransmission level for all data packets that cannot correspond to various traffic data in NAN. This consideration is especially important in smart grid applications, because data packets for different services have different sizes and various QoS requirements. The second problem is that ALM still does not address the load balancing and may route the traffic to a more congested area where nodes are highly loaded, which may introduces more flows into that path and increases the traffic load of forwarding nodes resulting in increasing the end-to-end delay as well as degrading the network throughput and reliability. Furthermore, even though different types of applications can be distinguished by EDCA, the fixed parameters in EDCA are unable to satisfy the various QoS demands of NAN. When the network load is heavy, the QoS of some highpriority traffic data cannot be guaranteed due to intense background channel contention for the fixed queue access mechanism of EDCA. And under low load condition, the low priority traffic needs long back-off time to access channel resulting in wasting the idle slot time, so that the resources utilization rate will be reduced.

Therefore, the main objective in this paper is to guarantee the various QoS requirements and increase the reliability of SG last smile communication by resolving above problems. To achieve this, we propose a NAN QoS-aware and load-balance routing scheme based on HWMP, named NQA-LB, which is suitable for special NAN in smart grid. NQA-LB not only considers the data packets size but also captures the accurate frame loss probabilities for various applications coming from MAC layer, which is capable of providing differentiated and efficient services for NAN applications with various priorities. In addition, the traffic load of forwarding nodes in a path is aware in NQA-LB for achieving load balance among transmission paths. In view of 
the disadvantages of EDCA in QoS guarantee and collision avoidance, we further present an EDCA based adaptive priority adjustment scheme (AP-EDCA) for NQALB, which dynamically adjusts packet's priority based on frame loss probability and queue length of each AC, to increase the network throughput under low load condition and improve the reliability of applications with high QoS requirement under heavy load condition. We evaluate the performance of NQA-LB and AP-EDCA on ns-3 in NAN scenarios with various applications. The simulation results show that our proposal satisfies the various requirements of NAN applications much better than original HWMP, and also the outperformance of our scheme in packet delivery ratio, end-to-end delay and throughput compared to some existing schemes.

The reminder of this paper is organized as follows. In section 2, we review the related work of the research area. Then, we propose the NQA-LB routing scheme in section 3 and detail the AP-EDCA in section 4. Simulation results are shown in section 5. Finally, section 6 concludes this paper.

\section{RELATED WORK}

Thus far, several approaches have been proposed by improving HWMP protocol for meeting QoS requirements and providing better transmission reliability for various services and applications in SG systems. A QoS mechanism called Optimized MultiConstrained Routing (OMCR) is proposed in [10], which analyzed the dynamics of power market and the impacts of communication metrics like delay and outage on the revenue of home appliances, the SG control center return one or more optional routing for home applications through sending QoS requests to guarantee the QoS requirements. In [11], Xu and Wang modeled the SG networks and presented the communication delay analysis in typical wireless mesh network deployment scenarios of SG with Voronoi diagram, which provides theoretical guiding rules for designing WMN to meet the delay requirements. Dabin et al added a delay-tolerant management module in HWMP routing maintenance mechanism so that the latency-tolerant data can be cached rather than discarded directly when links break down and sent after the routes are repaired in [12]. This mechanism guarantees high QoS requirements of important flows by scarifying end-to-end delay of delay-tolerant flows. In addition, the main issues about reliability of IEEE 802.11s in SG communication network are analyzed by Kim et al $[13,14]$ and they are improved by modifying routing metric, routing mechanisms and routing maintenance mechanism in HWMP. But, the load balance between paths have not considered in above approaches.

Besides optimizing routing metric, many studies tend to improve EDCA mechanism to improve the QoS of IEEE 802.11s based WMNs to satisfy the NANs QoS demands. Since in IEEE802.11e based WMNs, the transmission performance of traffic with low priority is poor because of the default parameters presented in EDCA scheme, so a cross-layer mechanism based on EDCA (CL-EDCA) is proposed to promote the QoS of legacy EDCA in [15]. CL-EDCA can dynamically adjust packet's priority of each flow at network layer by considering per AC delay and queue length. In order to meet various QoS requirements of NAN, Jaebeom et al presented a conten- 
tion mitigated EDCA algorithm called CM-EDCA in [16], which utilized the packet collision probability and MAC retransmission to change the packet priority and reduce packet collision. It is compatible with IEEE 802.11e and easy to implement for SG devices, while the poor HWMP routing metric is not considered.

Furthermore, the nature of routing algorithms in the NAN network may cause an inefficient use of network resources such that some of the gateways are heavily loaded while the others are lightly loaded. Therefore, many studies use multiple gateways to balance the traffic load between gateways. A tree-based mesh routing scheme based on multi-gateway mesh network architecture is presented in [17]. The multigateway mesh routing scheme is based on a flexible mesh network architecture that expands on the hybrid tree routing of the IEEE 802.11s. In [18], authors devised a load-balance scheme that distributes NAN data to multiple gateways. In the proposed scheme, a relay node probabilistically selects its serving gateways by explicitly considering the congestion levels of the gateways to distribute the loads of congested gateways to lightly loaded gateways fast. However, they neglected the HWMP routing metric ignoring the queuing delay of packets, which may introduce more flows into the path to light loaded gateway simultaneously, and thus increases the traffic load of the forwarding nodes. As a result, end-to-end delay is increased due to the growing length of buffer queues of intermediate nodes and average network throughput is degraded because more collision will happen in intermediate congested areas.

Therefore, based on the HWMP and EDCA, we aim to design a new NAN QoSaware and load-balance routing scheme for SG in this paper, aiming to select accurate transmission path for various applications with less load so as to meet QoS demands and improve the SG system performance in terms of reliability and the rate of network resources utilization.

\section{PROPOSED ROUTING SCHEME}

In SG environments, a NAN may provide various services to a varying number of applications which vary in data size and QoS requirements. For example, power control information and users on-demand responses information demand higher QoS requirement, they are also very important to SG for guaranteeing high efficiency of whole grid. In order to satisfy the requirements of QoS about various NAN applications, we differentiate various applications based on QoS requirements by employing EDCA scheme firstly. Them, we analyze the Markov model for EDCA to obtain the frame loss probability of specific NAN applications. Finally, we detail the HWMP based NAN QoS-aware and load-balance routing scheme (NQA-LB), which extends the airtime of HWMP by capturing the frame error rate and adding the queuing delay of each class of applications with different packets size separately combining with EDCA. 


\subsection{NAN Applications Differentiation}

EDCA defines four access categories (ACs) with different priorities: AC_VO, AC_VI, AC_BE and AC_BK represent voice, video, best effort and background respectively from high to low. Each AC has its own transmission queue and a set of channel access parameters to contend for transmission opportunities, they are minimum contention window $\left(C W_{\min }\right)$, maximum contention window $\left(C W_{\max }\right)$ and Arbitrary Inter-Frame Space (AIFS). If an AC has a smaller AIFS, $C W_{\min }$ and $C W_{\max }$, traffic in this AC has a better chance to access the channel earlier than ones of lower service classes. The contention window $C W_{i, j}$ for $i$ AC after $j^{\text {th }}$ transmission failure is calculated as follows:

$$
C W_{\mathrm{i}_{j} j}=\min \left[2^{j}\left(C W_{i}^{\min }+1\right)-1_{s} C W_{i}^{\max }\right], i \in 0_{s}{ }^{\prime}{ }_{x} N_{c}-1_{s} j \in 0, \cdots, m,
$$

where $N_{c}$ is the number of ACs with default value $4, m$ is the maximum retransmissions with 4 or 7 . Nodes can access after backing off channel until the channel measured idle time for an AIFS. The AIFS is defined in the EDCA as Fig. 2 and it is calculated as equation (2), where SIFS is the short inter-frame space.

$$
\text { AIFS }[A C]=\text { SIFS }+ \text { AIFSN }[A C] \times \text { SlotTime }
$$

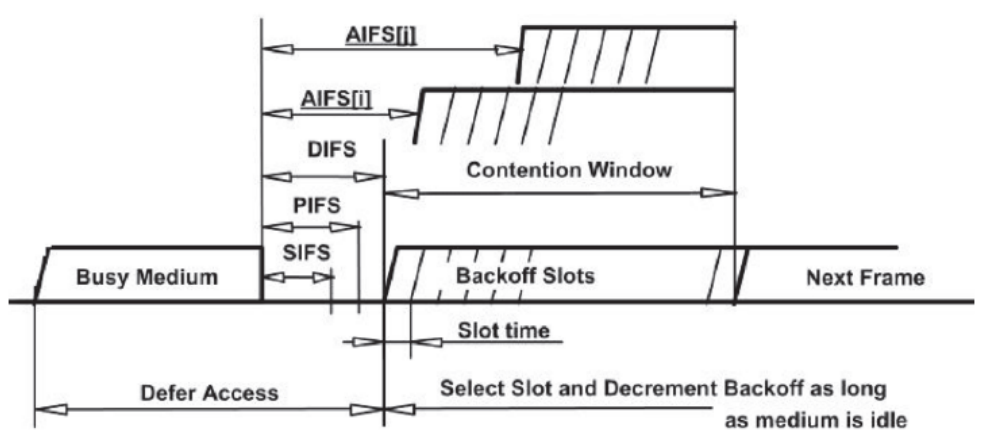

Fig. 2. AIFS defined in EDCA mechanism

Table 1. Smart grid Applications and EDCA priority

\begin{tabular}{ccllll}
\hline Priority & AIFSN & CW $_{\min }$ & CW $_{\max }$ & QoS & Application Type \\
\hline 3 & 2 & 7 & 15 & AC_VO & Control information \\
2 & 2 & 15 & 31 & AC_VI & $\begin{array}{l}\text { AMI demand response } \\
\text { Power quality data }\end{array}$ \\
1 & 3 & 31 & 1023 & AC_BE & $\begin{array}{l}\text { Periodic meter reading } \\
\text { data } \\
0\end{array}$ \\
\hline
\end{tabular}


Various traffic demand various QoS requirements in NAN, traffic such as control information and power quality monitoring data should be assigned with high priority for stable electric service of the SG system, while video surveillance data has low priority for guaranteeing the higher priority data even with strict latency. The mapping of NAN applications and EDAC priority are shown in Table 1.

\subsection{Model Analysis for EDCA}

As in [19], the Markov chain of priority $i(i=0,1,2,3)$ class for EDCA is shown as Fig.3. The model states consist of 3 variables $\left(i_{j}, j, k\right)$. The first variable $i$ denotes the priority of the transmitted packet. Let $p_{\mathrm{i}}$ denotes the probability that a transmitted frame collides and also equals to probability that a station in the back-off stage for priority $i$ class senses the channel busy. This has to be calculated separately for each priority class. The variable $j$ in the model represents the number of collisions that occurred when trying to transmit a packet. The last variable $k$ is the back-off counter of the stations.

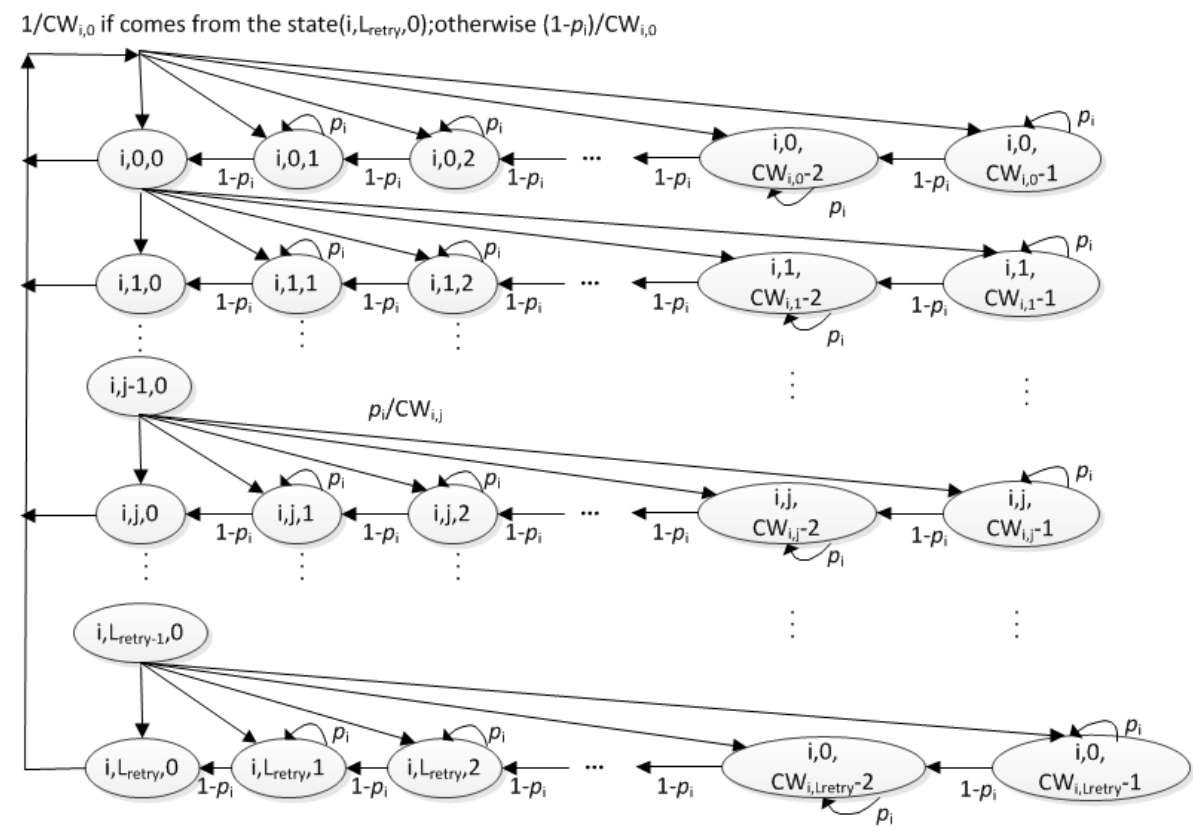

Fig. 3. Markov model for the priority class in EDCA

For a given station in the priority $i$ class $(i=0,1,2,3)$, the transition probabilities of the Markov model in Fig. 3 are listed as follows, where $L_{\text {yetry }}(i)$ is the number of retransmission allowed for $i$ class:

$$
\begin{aligned}
& P\left[\left(i_{i}, k\right) \mid(i, j, k+1)\right]=1-p_{i}, 0<k<C W_{i, j}-2,0<j<L_{\text {yetry }}(i) \\
& P[(i, j, k) \mid(i, j, k)]=p_{i}, 1<k<C W_{i, j}-1,0<j<L_{\text {retry }}(\mathrm{i})
\end{aligned}
$$




$$
\begin{aligned}
& P[(i, j, k) \mid(i, j-1,0)]=\frac{p_{i}}{\sigma W_{i, j}}, 1<k<C W_{i, j}-1,0<j<L_{\text {retry }}(i) \\
& P\left[(i, 0, k) \mid\left(i, L_{i, y e t r y}, 0\right)\right]=\frac{1}{C W_{i, 0}}, 0<k<C W_{i, 0}-1 \\
& P[(i, 0, k) \mid(i, j, 0)]=\frac{1-p_{i}}{C W_{i, 0}}, 0<k<C W_{i, 0}-1,0<j<L_{\text {yetry }}(i)
\end{aligned}
$$

Let $b_{i, j, k}=\lim _{t \rightarrow=0} P\left(i_{v} j_{y} k\right)$ be the stationary distribution of Markov chain that denotes the probability of the packet with priority $i$ where the collision encountered for $j^{\text {th }}$ and the back-off counter is equal to $k$. Then the following relations can be driven through chain regularities:

$$
\begin{aligned}
& b_{i, j, 0}=p_{i}^{j} b_{i_{0}, 0,0} \quad 0<j<L_{\text {retryt(i) }} \\
& b_{i, j, k}=\frac{\omega W_{i, j}-k}{\omega W_{i, j}} \frac{1}{1-p_{i}} b_{i, j, j} 0<j<L_{r e t r y}(i \mathfrak{i})^{x} 1<k<C W_{i, j}-1 \\
& \sum_{j=0}^{L_{\text {ratryy }}(i)} \sum_{k=0}^{C W_{i, j}-1} b_{i, j k}=1
\end{aligned}
$$

From (3), (4) and (5), we get

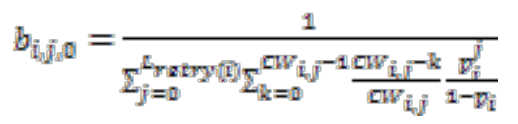

Let $\tau_{i}$ be the probability that a station in priority $i$ class transmits during a generic slot time. It is calculated by summing the steady state probabilities of states where the back-off counter is equal to 0 .

$$
\tau_{i}=\sum_{j=0}^{L_{\text {ratry(1) }}} b_{i, j, 0}=\sum_{j=0}^{L_{\text {ratry(d) }}} p_{i}^{j} b_{i, 0,0}=b_{i, 0,0} \frac{1-p_{i}^{L_{\text {ratryy }}}}{1-p_{i}}
$$

A transmitted frame collides when one more station also transmits during a slot time. So the probability $p_{i}$ that a station in the back-off stage for priority $i$ class senses the channel busy is given as:

$$
p_{\mathrm{i}}=1-\left(1-\tau_{\mathrm{i}}\right)^{\mathrm{n}_{\mathrm{i}}} \prod_{\mathrm{l}=0 \mathrm{i} \neq \mathrm{i}}^{\mathrm{a}}\left(1-\tau_{\mathrm{i}}\right)^{\mathrm{n}_{\mathrm{l}}}
$$

where $n_{i}(i=0,1,2,3)$ denote the number of stations in priority $i$ class. Let $p_{\operatorname{loss}(i)}$ denotes the frame loss probability for priority $i$ class. Transmission attempt will be $L_{\text {retryy(i) }}$ before losing packet. Hence, the frame loss probability $p_{\text {loss (i) }}$ and the frame success probability $p_{\text {suce }(i)}$ for priority $i$ class can be obtained as:

$$
p_{\text {losg }(\mathbb{Q})}=p_{\mathrm{i}}^{L_{\text {ratry }}(t)+1}
$$




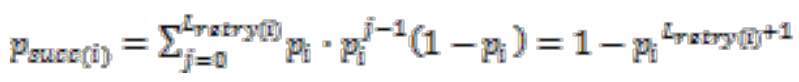

We can get the optimum transmission probability $\tau_{\text {opt }}$ that provides maximum achievable throughout according to paper [19], where $N$ is the number of ACs and $n$ is the number of stations.

$$
\tau_{\text {opt }}=\frac{\sqrt{n(2 n N+n-2 N)}-n}{\left(n^{2}-n\right) N}
$$

\subsection{NQA-LB Routing Scheme}

The default routing metric of HWMP is airtime link metric (ALM) to identify an efficient radio-aware path. The calculation of the ALM for each link is based on the following equation:

$$
C_{a}=\left(O_{c a}+O_{p}+\frac{B_{\varepsilon}}{\gamma}\right) \frac{1}{1-\theta_{f}}
$$

where $O_{c a}$ and $O_{p}$ are the channel access overhead and protocol overhead, $B_{t}$ is the test frame size, $r$ is the data rate, and $e_{f}$ is the test frame error rate. The draft does not specify how to calculate the frame error rate, leaving this choice to the implementation.

ALM reflects the amount of channel resources consume by transmitting test frame over a particular link, which is similar to ETT [20]. The left part in the brackets of (12) represents transmission time of a frame and the right part measures the number of transmission attempts needed to successfully deliver a packet. However, it is hard for ALM to meet QoS requirements very well when integrated into the smart grid network. Because using fixed test frame and same frame error rate calculation for all data packets in ALM cannot correspond to various traffic data with different priorities from NAN. Therefore, we modify the routing metric of HWMP as (13) by including actual packets size, and calculating frame error rate separately for various applications obtained from EDCA, subsequently the routing metric of HWMP can route more accurately for various NAN applications to meet the different QoS demands. In (13), $B_{(i)}$ is the packet size of applications with priority $i(i=0,1,2,3)$, and $\varepsilon_{f(i)}$ is the frame error rate of $i$ class which equal to frame loss probability ploss (i) calculated from equation (9).

$$
C_{a(1)}=\left(O_{c \alpha}+O_{p}+\frac{B_{(\mathrm{T})}}{\gamma}\right) \frac{1}{1-\theta_{f(\mathrm{l})}}
$$




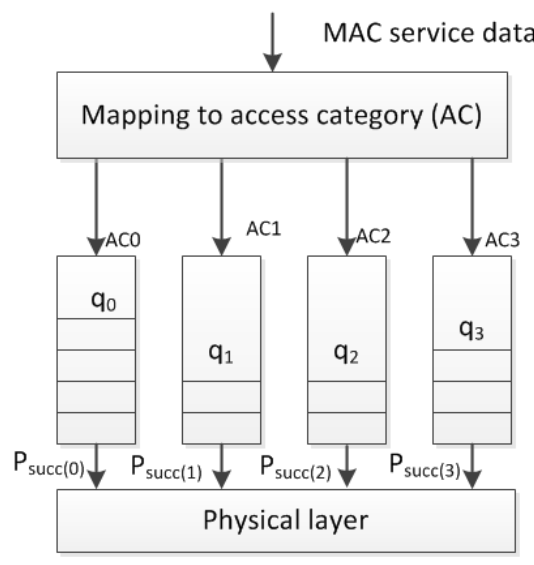

Fig. 4. EDCA basic queue access model

Furthermore, ALM ignores the queuing delay of packets which may route the traffic to a more congested area where nodes are highly loaded. This is because this metric always select the path with higher data rates, which may introduce more flows into that path and thus increase the traffic load of the forwarding nodes. As a result, endto-end delay is increased due to the growing length of buffer queues of intermediate nodes and average network throughput and reliability are degraded because more collision will happen in congested areas. Hence, we take consideration of queuing delay of different applications separately to balance traffic load between transmission paths, to decrease the congestion happened in intermediate nodes so as to improve the network performance. Queuing delay is defined as the period from the instant that a packet added to buffer to the instant that it is to be serviced (becoming the first row), which equals to the sum of transmission delay of all packets in queues transmitted before current packet being serviced. So the end-to-end delay of a packet of priority $\tilde{i}(i=0,1,2,3)$ class is expressed as:

$$
D_{\mathrm{i}}=D_{t(\mathrm{i})}+D_{Q(\mathrm{i})},
$$

where $D_{t(\mathbb{R})}$ is the transmission delay of packet with priority $i$ class that equal to ALM $\left(D_{t(i)}=C_{a(i)}\right)$ and $D_{Q(i)}$ is the queuing delay of current packet. The EDCA basic access model is shown as Fig. 4, each AC has its own transmission queue, where $p_{\text {suec }(\mathbb{i})}$ denotes the probability of a packet of class $i$ to be transmitted without being dropped, and it can be calculated from (10) in 3.2. If there are $q_{i}$ packets in queue of class $i$ and $q_{j}$ packets in queue of class $j(j \neq i)$ when adding a packet into queue of class $i$. We suppose that no packets are added into buffer of class $j$ in the calculation period, so the queuing delay of current packet can be in one of the following two situations:

(1) When packets in queue of class $i$ are smaller than that of class $j\left(q_{i}<q_{j}\right)$ : 


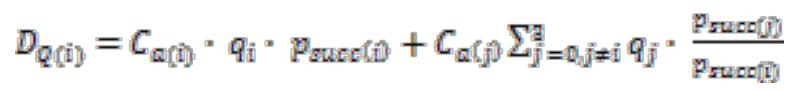

(2) Otherwise $\left(q_{i}>q_{j}\right)$ :

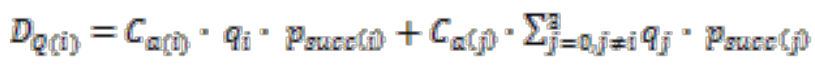

Therefore, we modify the ALM of HWMP as:

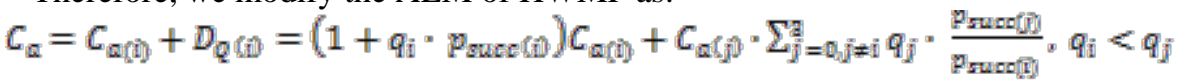

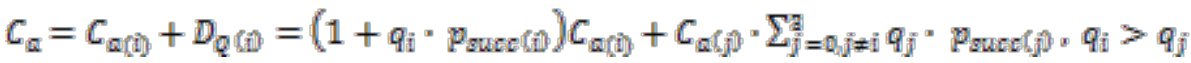

The calculation of $C_{a(\hat{i})}$ and $C_{a(j)}$ is shown in (13). Owning to the instantaneous queue length changes rapidly, frequent rerouting might be incurred if we directly utilize it to estimate the queuing delay. So we employ EWMP (Exponentially Weighted Moving Average) to reduce bias in order to prevent frequent rerouting, $\alpha$ is a set in the range of $[0,1]$ :

$$
q_{\mathrm{i}}=(1-\alpha) q_{\mathrm{i}}+\alpha q_{\mathrm{i} p r e}
$$

\section{ADPTIVE PRIORITY ADJUSTMENT SCHEME AP-EDCA}

Although the QoS requirements of various NAN applications can be satisfied by the NQA-LB, but since the fixed parameters in EDCA, the low priority traffic will take long back-off time to access channel which will result in reducing the resource utilization rate under low load condition. In addition, the reliability of NQA-LB will be decreased as a result from intense background channel contention under heavy load condition. Adjusting the EDCA parameters dynamically is propitious to wireless networks for supporting QoS, but modifications to the IEEE 802.11e standard cannot backward compatible with original EDCA. Therefore, we present an EDCA based adaptive priority adjustment scheme, called AP-EDCA, which dynamically adjusts packet's priority to increase the throughput under low load condition and to mitigate the collision under heavy load condition to improve the reliability of applications with high QoS requirements.

We put to use the sum of packet loss probability of four priority classes to indicate the network condition (NC) and it can be calculated as (19) based on (9). The EWMA is employed to calculate the average packet loss probability to include the historical packet loss probability, where $\beta$ is set in the range of $[0,1], N C_{c u r y}$ is the current packet loss probability and $N C_{p r e}$ is the historical average packet loss probability:

$$
N C=\sum_{i=0}^{\mathbb{a}} p_{\operatorname{loss}(\mathbb{i})^{3}, i} i=0,1,2,3,
$$




$$
N C=(1-\beta) N C_{c u r r}+\beta N C_{p r e}
$$

The traffic load condition (TC) is calculated by using the average queue length of each AC. Each node traces the queue length of each AC in period and the calculation of TC is similar to (19) and (20), where $q_{\mathrm{i}}$ is the average queue length of class $i$ and $q_{\mathfrak{i}_{(\max )}}$ is the maximum queue level of queue $i$ :

$$
\begin{aligned}
& T C[i]=\frac{q_{i}}{q_{i}(\max )}, i=0,1,2,3, \\
& T C[i]=(1-\gamma) T C[i]_{c u r y}+\gamma T C[i]_{p y e}
\end{aligned}
$$

As shown in algorithm 1, Nodes analyze the network condition $N C$ and each AC traffic load $T C[i]$ as well as compare them with preset threshold values, then dynamically adjusts the priority of packets according to comparison results. When the $N C$ is lower than threshold value, that shows the network load is light. Similarly, if the queue length of high-priority traffic (AC3, AC2) is less than minimum threshold value, that indicates network resource has a surplus. Hence we give high priority services to low priority packets so that more chances are given to access channel to improve the utilization of the channel resources. If the network condition is moderate, the priority of each flow is unchanged and packets are sent in accordance with the original EDCA access mechanism. When the NC is higher than threshold value, it shows network congestion is serious resulting in that high QoS traffic cannot be guaranteed. If the queue lengths of lower ACs (AC0, AC1) are smaller than minimum threshold value as well as the high-priority (AC2, AC3) queue lengths are larger than maximum threshold value at this point, the priority of the high priority packets should be lowered. By choosing the queue which has lower priority, packets can choose their contention window from a wider range to avoid collision and in consequence the network reliability is improved.

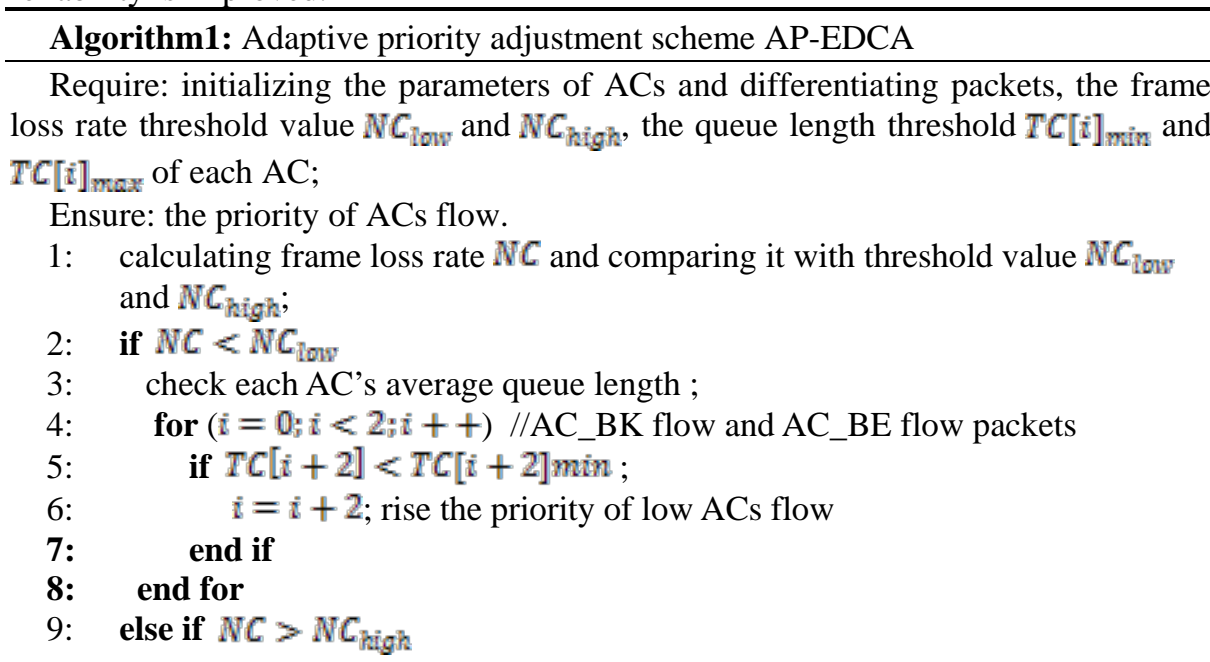




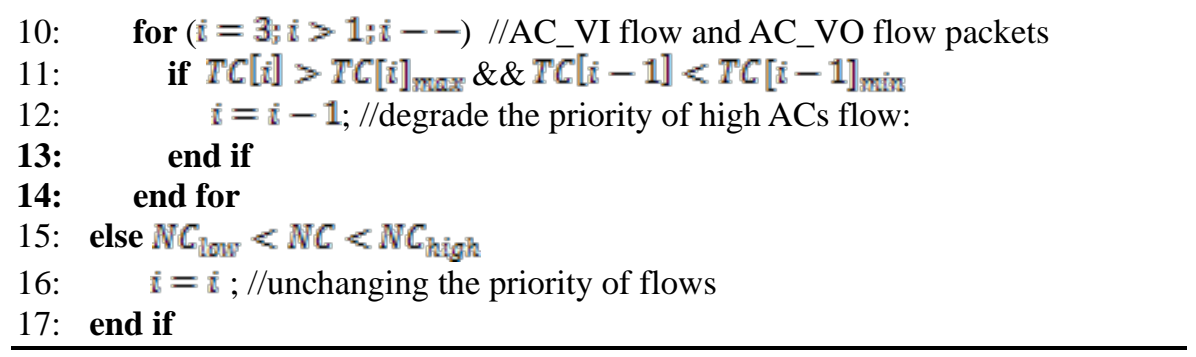

\section{SIMULATION AND ANALYSIS}

\subsection{Simulation Environment}

Since the ns3 platform includes IEEE 802.11s model and HWMP routing protocol, which realized the EDCA mechanism in MAC layer, we evaluate the performance of our proposed NQA-LB on ns3-19 by modifying IEEE 802.11s codes in mesh model and EDCA codes in wifi model under a NAN environment. In simulation scenarios, nodes are laid out on the network in a grid topology with node sizes (NS) distributed from 9 to 64. We also add an extra mesh node into network as a gateway outside interferences to gather the traffic of rest mesh nodes. The distance between each mesh STA is $150 \mathrm{~m}$. Each mesh STA is equipped with one 802.11a transmission device with a maximum transmission rate of $54 \mathrm{Mb} / \mathrm{s}$. And the AARF is used as rate adaptive adjustment algorithm. The factors $\alpha, \beta$ and $\gamma$ are set to 0.5 . The transmission probability of each station is the same and calculated according to equation (11). Other simulation parameters are shown in Table 2. For each simulation scenario, we repeat simulation for 10 runs with different random seeds and take the average.

Table 2. Simulation environment

\begin{tabular}{ll}
\hline Parameter & Value \\
\hline Simulation time & $500 \mathrm{~s}$ \\
PHY Standards & IEEE 802.11a \\
Rate adaptive & enable (AARF) \\
HWMP RANN interval & $5 \mathrm{~s}$ \\
$q_{\mathrm{i}}$ & 50 (pax \\
$N C_{\text {low }}$ & $0.01 / 0.1$ \\
$T C[0,1]_{\min }$ & $0.3 / 0.7$ \\
$T C[2,3]_{\min }$ max & $0.2 / 0.8$ \\
EDCA parameters & default \\
\hline
\end{tabular}

NAN applications are configured and differentiated according to [21] and SG Network System Requirements Specification v4.1-draft3 [22]. Power management data and AMI management data require high reliability to stable the whole SG so the priorities are set to 3 . While the lowest priority is set to video surveillance data since they are not important in SG compared with other data even with strict delay require- 
ment, and detail parameters are shown in Table3. AMI data can be thought as data from HAN nodes, whereas power quality, power management and video surveillance data come from the NAN applications. Periodic AMI, AMI management, periodic power quality, and power management data are reported to the root at every specified interval and all applications are generated with the gateway (root mesh STA) as their destination.

Table 3. Smart grid applications setting

\begin{tabular}{|c|c|c|c|c|}
\hline Type of service & $\begin{array}{l}\text { Transmis- } \\
\text { sion interval }\end{array}$ & $\begin{array}{l}\text { Application } \\
\text { size }\end{array}$ & $\begin{array}{l}\text { Priority } \\
\text { (EDCA) }\end{array}$ & $\begin{array}{l}\text { Requirement } \\
\text { latency }\end{array}$ \\
\hline Periodic AMI & $15 \mathrm{~s}$ & 123 bytes & 1 & $<15 s$ \\
\hline AMI management & $300 s$ & 4000 bytes & 3 & $<1 \mathrm{~s}$ \\
\hline Periodic power quality & 3s & 3000 bytes & 1 & $<3 s$ \\
\hline Power management & $300 s$ & 4000 bytes & 3 & $<1 \mathrm{~s}$ \\
\hline Requested AMI & On-demand & 123 bytes & 2 & $<5 s$ \\
\hline Requested power quality & On-demand & 2000 bytes & 2 & $<5 s$ \\
\hline Video surveillance & Constant & $250 \mathrm{kB} / \mathrm{s}$ & 0 & $<100 \mathrm{~ms}$ \\
\hline
\end{tabular}

\subsection{Performance Evaluation of NQA-LB}

In the first experiment, we compare the performance of our proposed routing protocol NQA-LB with original HWMP and HWMP-reliability enhancement routing scheme (HWMP-RE) proposed in [13] in different mesh network sizes. The MAC layer schemes of all protocols are original EDCA IEEE 802.11s used default. The amount of traffic generated in this environment naturally causes congestion in the network when the number of nodes gradually increases.

Simulation results are shown in Figs. 5 to 7 below. Fig. 5 shows the packet delivery ratio (PDR) of three protocols that represents the ratio of total number of packets received by the root nodes and the overall number of packets transmitted by all NAN mesh nodes in the network. With the number of nodes increasing, network becomes increasingly congested and PDR declines in this figure. Owing to poor route selection, the decline of ratio in original HWMP is steeper than in other schemes. However, with the increase of traffic load, NQA-LB provides a higher delivery ratio by capturing the buffered queue length of each forwarding node and selects a path with lower frame error rate and lightly loaded nodes for various applications. Therefore, it shows that our proposal compared with original IEEE 802.11s improves about 15\% and guarantees $\mathrm{PDR} \geq 80 \%$ when NS $\leq 64$. The HWMP-RE unable to aware traffic loads in the forwarding nodes even though the delay-tolerant model can reduce the packets loss probability when network is congested. Consequently, the performance of HWMP-RE is inferior to that of NAQ-LB.

The average end-to-end delay, which represents the average time required for a packet to reach root mesh STA from a source node (a mesh STA), is shown in Fig. 6. Since the optimized routing criterion which takes actual frame error rate and queuing delay into account during the path selection for various applications in NQA-LB, it 
could choose a better route to achieve a lower end-to-end delay even in the congested network. While, when 49 nodes are deployed in the network for HWMP-RE, the delay-tolerant management model begins to handle the delay-tolerant applications instead of being discarded when delay-tolerant packets cannot be transmitted timely, which actually increases the end-to-end delay of delay-tolerant applications, but this comes at the cost of much higher transmission reliability.

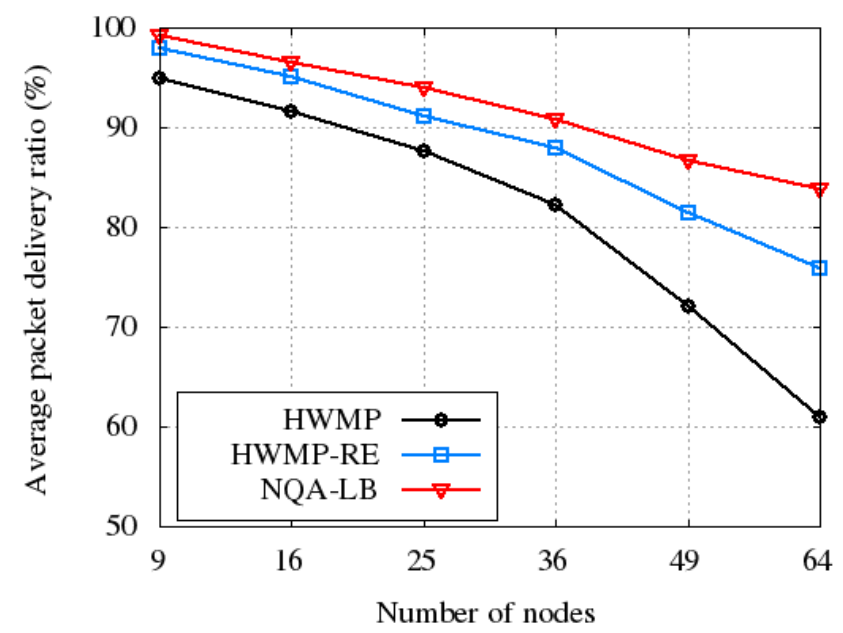

Fig. 5. Average packet delivery ratio comparison of three protocols in various network sizes

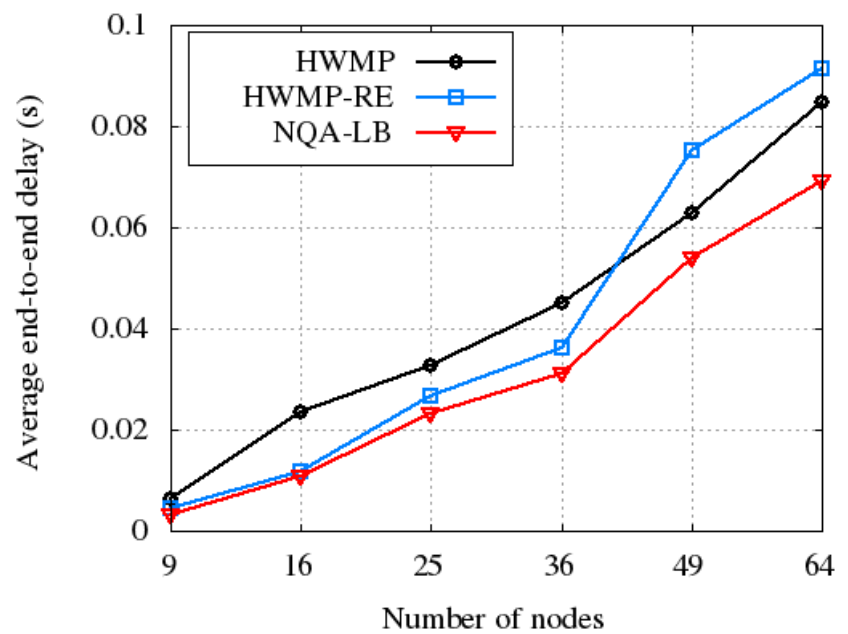

Fig. 6. Average end-to-end delay comparison of three protocols in various network sizes

Fig.7 shows the average throughput of three protocols which indicates the total amount of data received by root mesh STA in each second. The more the number of nodes, the more the data will be generated and this leads to an increase in the 
throughput. After a certain saturation NS=36, throughput of HWMP decreases because of insufficient resources. Since HWMP-RE and NQA-LB optimized the ALM for choosing a better transmission path for certain traffic, the throughput of both of them is still increased when NS $\geq 49$. Moreover, as NQA-LB could aware the number of packets in each AC queue. It is capable of selecting the better routes with less load, aiming to reduce the transmission collision and the overhead for retransmitting packets. By doing so, NQA-LB gets higher throughput than HWMP-RE when the network is congested.

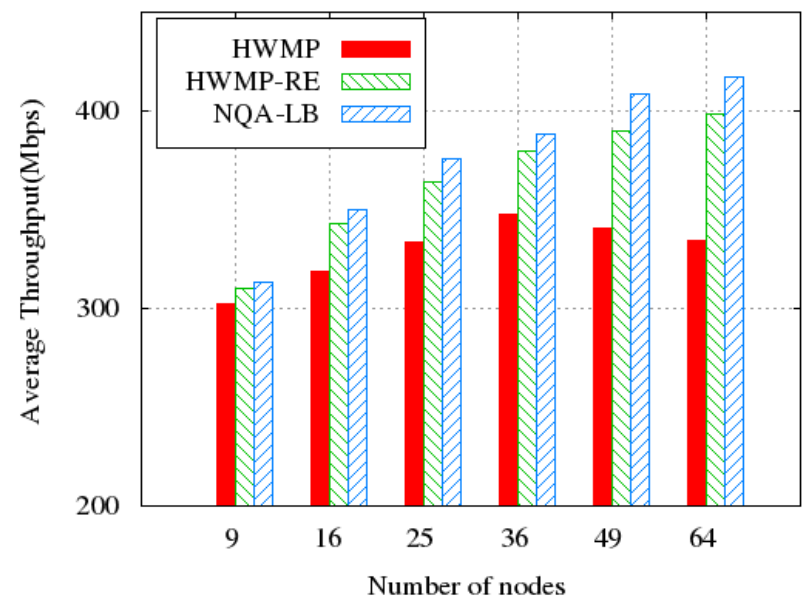

Fig. 7. Average throughput comparison of three protocols in various network sizes

\subsection{Performance Evaluation of AP-EDCA}

In the second experiment, we evaluate the performance of our adaptive priority adjustment scheme AP-EDCA comparing with original EDCA and CM-EDCA mechanism proposed in [16] based on the default routing protocol HWMP of 802.11s.

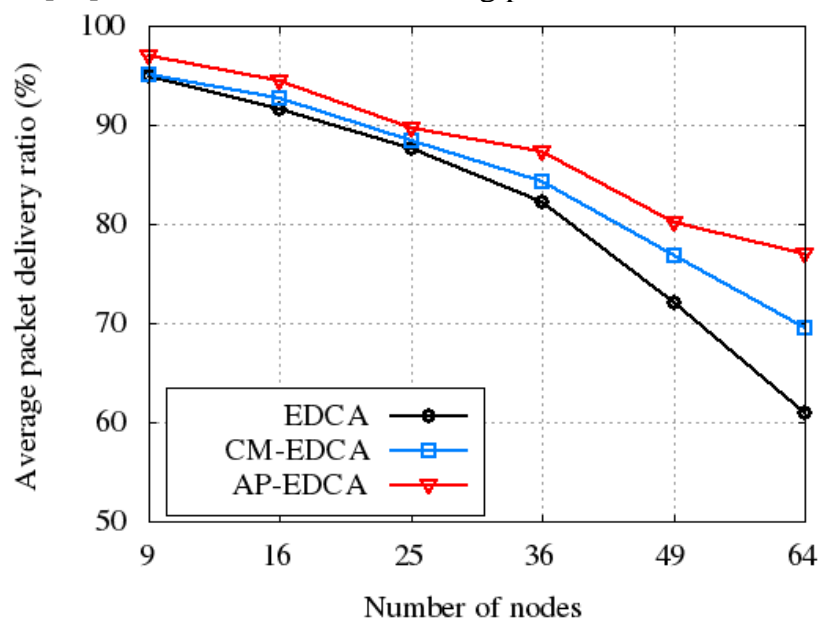


Fig. 8. Average packet delivery ratio comparison of three schemes in various network sizes

The average packet delivery ratio comparison of three schemes is shown in Fig.8. In this figure, the end-to-end packet reliability of AP-EDCA has higher compared to IEEE 802.11s using EDCA and CM-EDCA, because we adjust packets priority dynamically in MAC layer according to network state and each AC traffic load to speed the transmission of lower priority when network resources are abundant and reduce the congestion when network is congested. Even though CM-EDCA improves the reliability to a certain extent through changing packet priority and reducing packets collision when networks are congested, it does not consider the queuing length which may introduce more flows into the lower priority queues result in decreasing the performance of traffic with low priority, so the increasing of average PDR is not obvious.

Fig. 9 shows the average end-to-end delay comparison of three schemes in various network sizes. The average end-to-end delay of AP-EDCA decreases greatly comparing with the other two schemes. This is because AP-EDCA raises the priority of lower AC packets to speed them that could decrease the end-to-end delay when network load is light; it also reduces the contention and congestion by balance the load between different priority queues in nodes when network is congested. In CM-EDCA, packets may select a lower priority queue when network is congested, which in turn increase the delay as lower priority have larger $\mathrm{CW}$ value, but the transmission collisions can be reduced hence the end-to-end delay on the whole is decreased compared to EDCA.

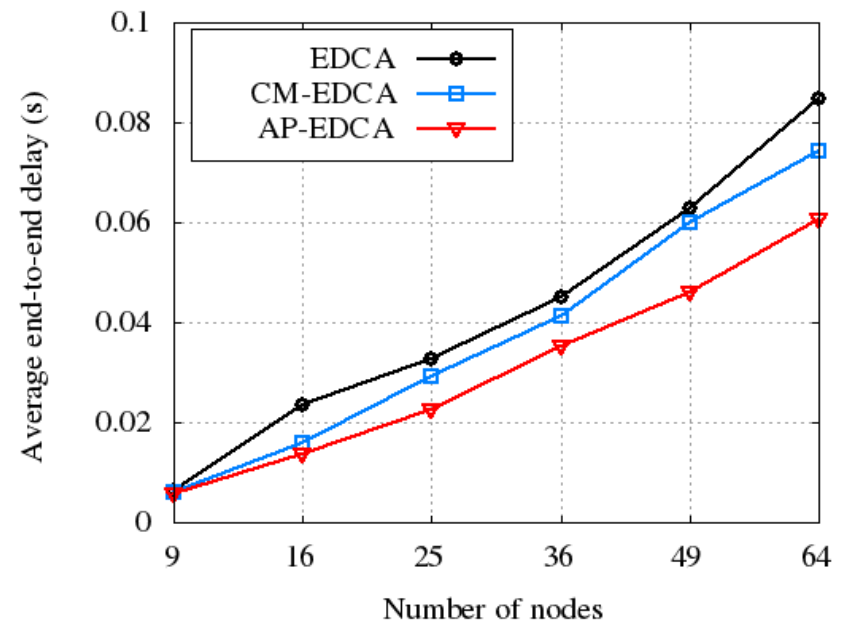

Fig. 9. Average end-to-end delay comparison of three schemes in various network sizes

\subsection{Protocol Comparison in Various Applications}

In this experiment, we compare the performance of our proposed scheme NQALB+AP-EDCA with HWMP, CM-EDCA and HWMP-RE in various NAN applications. Fig. 10 to 13 compare the packet delivery ratio of four schemes in various NAN 
applications (AMI and power management, Requested AMI and power quality, Periodic AMI and power quality, Video surveillance). From Fig.10, we see that the PDF of all applications in HWMP is almost similar and declines rapidly with the increasing number of nodes because it does not distinguish various applications. The PDF of all applications in CM-EDCA are close to that in HWMP when NS $\leq 36$. Network congestion starts to occur when NS $>36$, CM-EDCA alleviates the congestion by priority adjustment scheme, so the PDF of all applications increased 8\% comparing with HWMP. However, CM-EDCA cannot guarantee the QoS of important applications since it does not optimize metric for various NAN applications. We note that for HWMP-RE, the performance of video surveillance data transmission does not improve relative to CM-EDCA when network is congested, this is due to the delaytolerant traffic management, dropping the video surveillance data while retaining higher priority data for more reliable transmission. Since the NQA-LB not only considers the data packets size and captures the accurate frame loss probabilities for various applications coming from MAC layer, but also aware the traffic load of forwarding nodes to select less load paths for traffic, which is capable of providing differentiated and efficient services for NAN applications with various priorities. Besides, the AP-EDCA adjusts the priority of packets dynamically to speed packets transmission under light traffic load and decrease continues congestion under heavy load. Hence, the PDF of all applications is above $90 \%$ when NS $\leq 25$ and is considerably increased compare to CM-EDCA and HWMP-RE when network severely congested.

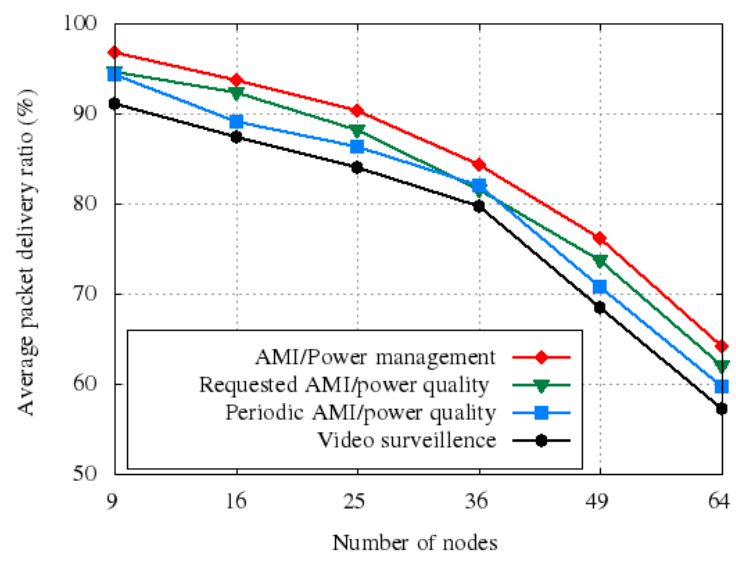

Fig. 10. Packet delivery ratio of HWMP in various NAN applications 


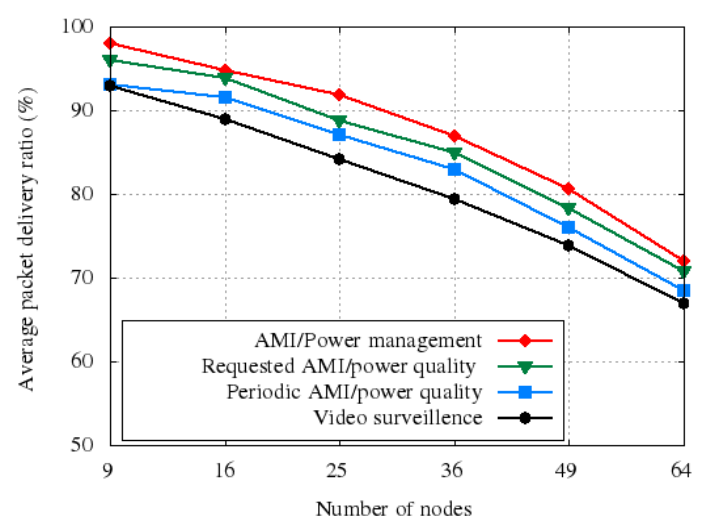

Fig. 11. Packet delivery ratio of CM-EDCA in various NAN applications

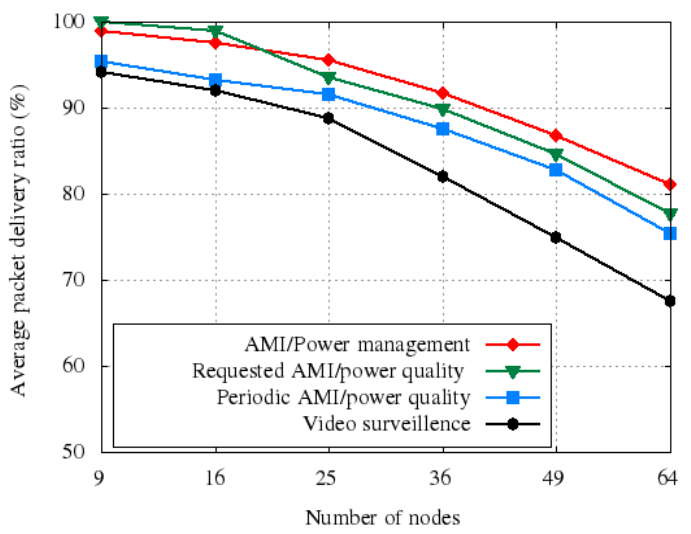

Fig. 12. Packet delivery ratio of HWMP-RE in various NAN applications

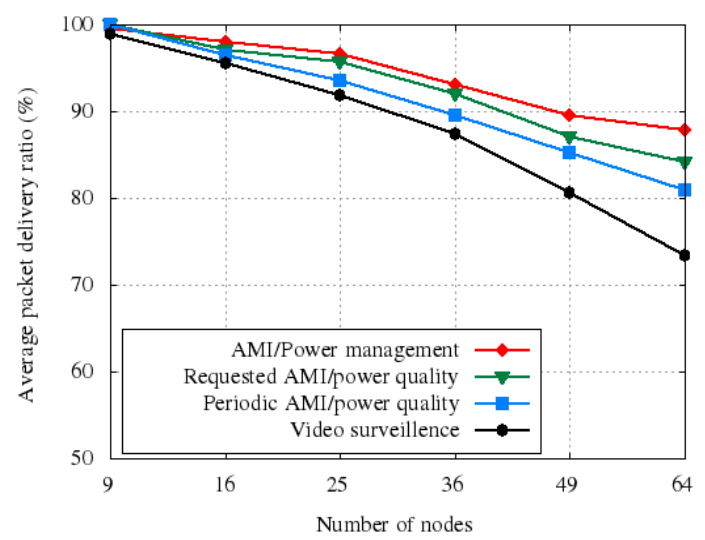

Fig. 13. Packet delivery ratio of NQA-LB+AP-EDCA in various NAN applications 
The end-to-end delay comparison for four schemes in various NAN applications are shown in Fig. 14 to 17. We can see from Fig. 14, the end-to-end delay of all applications in HWMP is similar in that all NAN applications transmitted with the same level. In CM-EDCA, owing to the selection of lower priority queues when network congestion occurs, the delay of some traffic is higher than HWMP. We can see that the delay of periodic power quality data is considerably increased, but much less than the requirement in SG. And for some traffic the delay is actually less than in EDCA scheme for less contention and collision in CM-EDCA which speeds up the transmission. When network is congested (NS>36), HWMP-RE handle delay-tolerant applications (Requested AMI/power quality data, Periodic AMI/power quality data) to support high reliability, so the end-to-end delay of delay-tolerant is increased rapidly with the increasing number of nodes. As shown in Fig. 12 and Fig. 16, HWMP-RE improves the reliability of SG by scarifying the delay of delay-tolerant applications. Comparing to other schemes, the delay of all applications in our scheme is greatly lower than in other three schemes. Therefore, NQA-LB + AP-EDCA guarantee the QoS for all traffic flows, at the same time, ensures the fairness of all traffic flows. This is owing to, NQA-LB gets packets loss probability of different flows timely from MAC layer to provide accurate route for particular traffic, and it considers the queuing delay of data packets that can dynamically schedule packets to the path with less load and less expected transmission delay. Besides, AP-EDCA adjusts packets priority dynamically in MAC layer according to network state and each AC traffic load to speed the transmission of lower priority when network resources are abundant and reduce the congestion when network is congested.

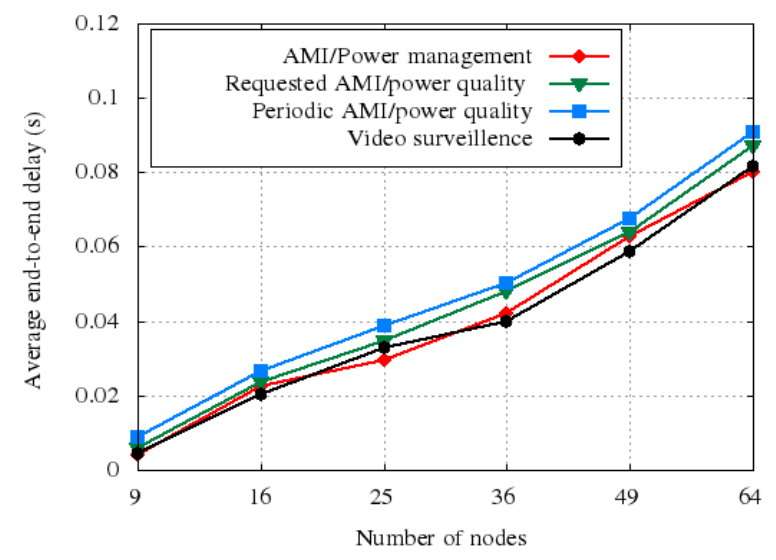

Fig. 14. End-to-end delay of HWMP in various NAN applications 


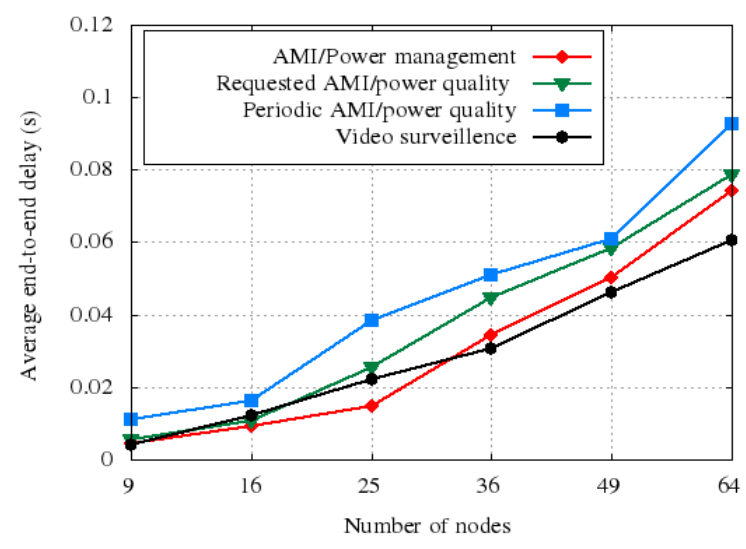

Fig. 15. End-to-end delay of CM-EDCA in various NAN applications

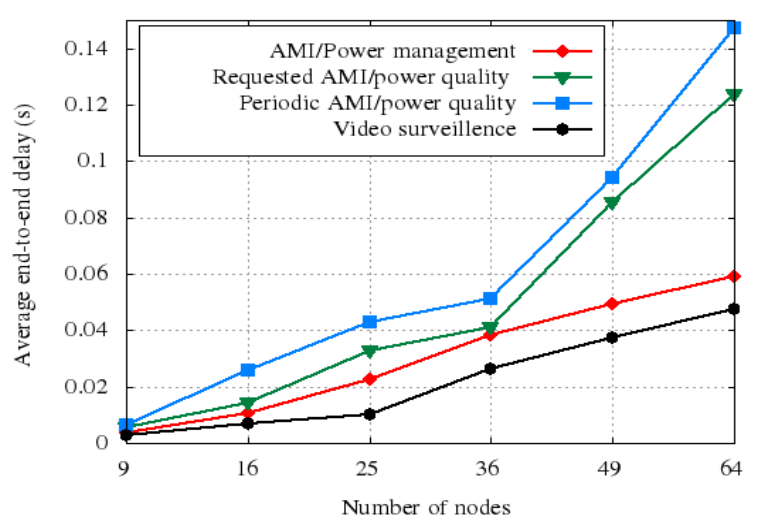

Fig. 16. End-to-end delay of HWMP-RE in various NAN applications

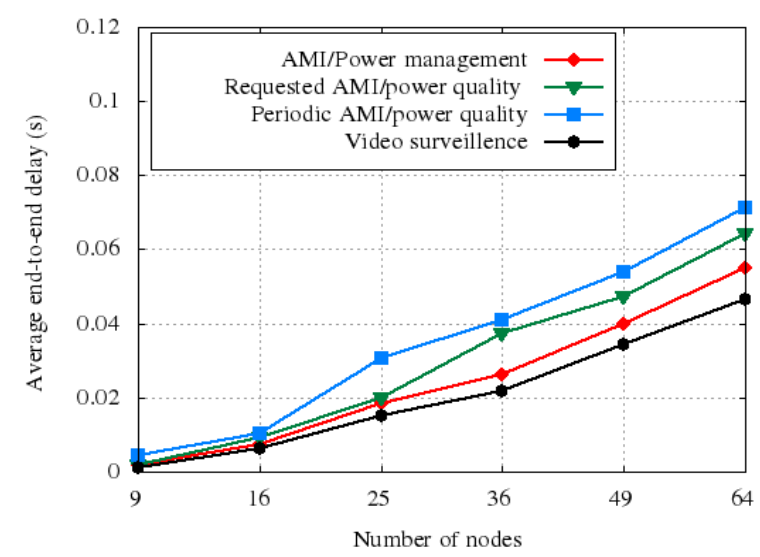

Fig. 17. End-to-end delay of NQA-LB+AP-EDCA in various NAN applications 
Overall, our NQA-LB and AP-EDCA not only can provide sufficient differentiated services for various NAN applications, but also can provide highly reliable wireless data transmission and satisfy various QoS requirements for smart grid environments by making optimizations to the original IEEE 802.11s HWMP and EDCA.

\section{CONCLUSION}

One of the most important challenges to implement smart grid communications is to provide a reliable and efficient last smile communications that covers the connectivity from the meters to the advanced metering infrastructure (AMI) head-end. In order to satisfy the various QoS demands and provide high reliable transmissions for NAN applications in smart grid, in this paper, we have first distinguished the various NAN applications by EDCA according to their QoS requirements regarding service differentiation. Then, a NAN QoS-aware and load-balance routing scheme (NQA-LB) has been proposed. The main contribution of the NQA-LB is that it has addressed the inadequacies of IEEE 802.11s in smart grid communications systems by using three methods. Simulation results on ns3 have shown that our proposal can satisfy NAN QoS requirements well and also significantly improved the average packet delivery ratio and throughput with a reduced end-to-end delay of all NAN applications, comparing to other existing schemes.

\section{Acknowledgement}

The author gratefully acknowledges support from National Natural Science Foudation of China projects of grant No. 61272149, 61379058, 61379057, 61350011 and JSPS KAKENHI Grant Number 26730056, 15K15976 and JSPS A3 Foresight Program.

\section{References:}

1. Fan, Z. and P. Kulkarni, et al. Smart Grid Communications: Overview of Research Challenges, Solutions, and Standardization Activities. IEEE Communications Surveys \& Tutorials, 15 (1): 21-38, 2013.

2. Fang, X. and S. Misra, et al. Smart Grid - The New and Improved Power Grid: A Survey. IEEE Communications Surveys \& Tutorials, 14 (4): 944-980, 2012.

3. Draft NIST Framework and Roadmap for Smart Grid Interoperability Standards, Release 3.0. 2014.

4. Meng, W. and R. Ma, et al. Smart Grid Neighborhood Area Networks: A Survey. IEEE Network, 28 (1): 1, 2014.

5. Zheng, L. and S. Parkinson, et al. Energy efficient communication networks design for demand response in smart grid. IEEE: 1 - 6, 2011.

6. Zheng, L. and N. Lu, et al. Reliable Wireless Communication Networks for Demand Response Control. IEEE Transactions on Smart Grid, 4 (1): 133-140, 2013. 
7. Akyildiz, I. F. and X. Wang, et al. Wireless mesh networks: a survey. Computer Networks, 47 (4): 445-487, 2005.

8. IEEE 802.11s, Part 11: Wireless LAN Medium Access Control (MAC) and Physical Layer (PHY) specifications Amendment 10: Mesh Networking, 2011.

9. Information technology- Telecommunications and information exchange between systemsLocal and metropolitan area networks- Specific requirements- Part 11: Wireless LAN Medium Access Control (MAC) and Physical Layer (PHY) Specifications. IEEE: i - 513.

10. H, L. and Z. W. QoS Routing in Smart Grid. Global Telecommunications Conference (GLOBECOM 2010): 1-6, 2010.

11. Xu, Y. and W. Wang. Wireless Mesh Network in Smart Grid: Modeling and Analysis for Time Critical Communications. IEEE Transactions on Wireless Communications, 12 (7): 3360-3371, 2013.

12. Dabin, K. and K. Jaebeom, et al. Developing the IEEE 802.11s based reliable Smart Grid mesh networks. Sensor, Mesh and Ad Hoc Communications and Networks (SECON), 2012 9th Annual IEEE Communications Society Conference on, Seoul: 88-90, 2012.

13. Kim, J. and D. Kim, et al. Improving the Reliability of IEEE 802.11s Based Wireless Mesh Networks for Smart Grid Systems. Journal of Communications and Networks, 14 (6): 629639, 2012.

14. Ji-Sun, J. and L. Keun-Woo, et al. Improving IEEE 802.11s Wireless Mesh Networks for Reliable Routing in the Smart Grid Infrastructure. Communications Workshops (ICC), 2011 IEEE International Conference on, Kyoto, 2011.

15. FU, Q. and Z. CHEN, et al. A Cross-layer QoS Scheme based on EDCA in Multi-hop Wireless Mesh Networks. Journal of Computational Information Systems, 2 (10): 817825, 2014.

16. Jaebeom, K. and L. Keun-Woo, et al. Contention mitigated EDCA algorithm for reliable smart grid mesh networks. Sensor, Mesh and Ad Hoc Communications and Networks (SECON), 2012 9th Annual IEEE Communications Society Conference on, Seoul, 48-50, 2012.

17. Gharavi, H. H. B. Multigate mesh routing for smart Grid last mile communications, IEEE: 275 - 280, 2011.

18. Park, J. and Y. Lim, et al. A scalable load-balancing scheme for advanced metering infrastructure network. RACS, ACM: 383 - 388, 2012.

19. Cil, M. Akar and E. Anarim. Adaptive optimization of EDCA parameters for improved QoS in multimedia applications," TURKISH JOURNAL OF ELECTRICAL ENGINEERING AND COMPUTER SCIENCES, 20(2) 1369-1388, 2012.

20. Draves, R. and J. Padhye, et al. Routing in multi-radio, multi-hop wireless mesh networks. MobiCom, ACM: 114 - 128, 2004.

21. Budka, K. C. and J. G. Deshpande, et al. Communication network architecture and design principles for smart grids. Bell Labs Technical Journal, 15 (2): 205-227, 2010.

22. OpenSG users group, SG network system requirements specification v4.1-draft3, http://www.osgug.ucaiug.org. 